\title{
Synthesis and Characterization of a Chitosan/PVA Antimicrobial Hydrogel Nanocomposite for Responsive Wound Management Materials
}

Samantha J. Rinehart ${ }^{1,2}$, Thomas D Campbell ${ }^{2}$, Kevin J. Burke ${ }^{1}$, Bianca Garcia ${ }^{1}$, Amy Mlynarski ${ }^{1}$, Samantha J. Brain ${ }^{1}$, Julianne M. Truffa ${ }^{1}$, James Rago², William E. Chura² and Jason J Keleher ${ }^{1 *}$

${ }^{1}$ Department of Chemistry, Lewis University, One University Parkway, Romeoville, IL 60446- 2200, USA

${ }^{2}$ Department of Biology, Lewis University, One University Parkway, Romeoville, IL 60446- 2200, USA

\begin{abstract}
The development of novel wound management materials must address several challenges to be most efficacious. Successful wound management should include the ability to adhere to wound surfaces, absorb wound exudates, and enhance bactericidal effectiveness. Few current biomaterials address all of these characteristics and in addition often have poor structural rigidity leading to a lack of protection and support to ensure proper cell growth. The focus of this study was to develop a multifunctional biomimetic nanocomposite system that will improve mechanical stability, effectively eliminate $E$. coli and S.aureus biofilm propagation, and serve as a scaffold for effective adult human dermal fibroblast (HDFa) growth. Synthetic parameters, such as the ratio of chitosan to PVA concentration and deposition method for the incorporation of $\mathrm{Ag}^{+}$-functionalized nanoparticles into the final nanocomposite, were optimized. It was determined the deposition reaction $\mathrm{pH}$ and $\mathrm{Ag}^{+}$concentration played a key role in the control of post reaction particle size and zeta potential. It was also determined that the concentration of $\mathrm{Ag}^{+}$functionalized nanoparticle incorporated into the nanocomposite must be greater than $5 \mathrm{mM}$ to achieve optimal control of biofilm formation. The prepared hydrogel nanocomposites demonstrated effective bacterial inhibition when exposed to cultures of $E$. coli, S. aureus, and MRSA. Lastly, human dermal fibroblast test results revealed that when PVA concentration increased in the system, there was no appreciable cell growth. On the other hand, when the ratio of biomimetic chitosan and PVA is optimized to reduce the overall amount of synthetic polymer (PVA), there was healthy cell growth. These results directly speak to a key finding that reveals the importance of the correct balance or the removal of synthetic polymer from the system.
\end{abstract}

Keywords: Biomimetic; Hydrogel; Chitosan; Nanoparticle; Nanocomposite; Antimicrobial; E. coli; S. aureus; MRSA; Human Dermal Fibroblast; tissue cell growth

\section{Introduction}

Hydrogels are networks of hydrophilic polymers that form welldefined three dimensional structures that are ideal for modification and functionalization [1]. The water insolubility of hydrogels and high swelling capability has led to their use in the design of biomaterials for applications such as tissue engineering, drug release systems, and wound management materials [2-8]. Hydrogels made from natural biopolymers are ideal for biomaterial applications owing to their intrinsic properties such as non-cytotoxicity and biodegradability $[9,10]$. Chitosan, which is a biopolymer produced as a byproduct of crustacean shells, is widely used as a backbone of hydrogels for such applications. In addition to the aforementioned properties, chitosan is often sought out for use in antimicrobial hydrogels for its inherent antibacterial property $[11,12]$. Although there has been extensive work highlighting the advantages of chitosan hydrogels, there are still some functional limitations.

The use of chitosan alone has shown to limit the mechanical strength of the hydrogel during swelling [13]. It has been reported that polymer blending can be used to improve physicochemical properties such as mechanical strength [14-16]. For example, Yang et al reported that chitosan based hydrogels cross-linked with polyvinyl alcohol (PVA) showed an increased mechanical strength compared to their chitosan only counterparts [14]. This and related work has led to the development of structurally stable hydrogels for many applications.

In recent years, significant attention has been focused on the use of chitosan based hydrogels for burn wound management [1719]. Chitosan-based hydrogels are biocompatible, can maintain a moist healing environment that provides a scaffold for cell growth while acting as a protective barrier from microorganisms to prevent secondary infections. Furthermore, they have shown to assist in absorbing excess wound exudates, meeting the requirements of an ideal wound dressing [19-21]. In efforts to increase structural rigidity and improve the antimicrobial efficiency (AME) without compromising the biocompatible integrity of these hydrogels, many studies have focused on the integration of antimicrobial silver $(\mathrm{Ag})$ and $\mathrm{Ag}$ functionalized semiconductor particles into their matrices $[22,23]$.

Ionic Ag and Ag-based compounds have been widely accepted as bacterial growth inhibitors since the 19th century due to their notable biocidal properties against several types of bacteria, including Escherichia coli and Staphylococcus aureus [24-26]. However, $\mathrm{Ag}^{+} \mathrm{can}$ be difficult to uniformly distribute throughout the hydrogel matrix. Therefore, Ag coated semiconductor particles offer an alternative incorporation approach. When a solid support, such as titanium dioxide $\left(\mathrm{TiO}_{2}\right)$, functionalized with $\mathrm{Ag}$ is placed in a suspension, the $\mathrm{Ag}^{+}$released from the surface can kill bacteria [27,28]. This allows for the gradual release of $\mathrm{Ag}^{+}$, which has shown to increase the longevity

*Corresponding author: Keleher J, Department of Chemistry, Lewis University One University Parkway, Romeoville, IL 60446-2200, USA, Tel: +1 815-838-0500; E-mail: KeleheJa@lewisu.edu

Received January 23, 2016; Accepted February 18, 2016; Published February 25, 2016

Citation: Rinehart SJ, Campbell T, Burke KJ, Garcia B, Mlynarski A, et al (2016) Synthesis and Characterization of a Chitosan/PVA Antimicrobial Hydroge Nanocomposite for Responsive Wound Management Materials. J Microb Biochem Technol 8: 065-070. doi: 10.4172/1948-5948.1000264

Copyright: (c) 2016 Rinehart SJ, et al. This is an open-access article distributed under the terms of the Creative Commons Attribution License, which permits unrestricted use, distribution, and reproduction in any medium, provided the original author and source are credited. 
Citation: Rinehart SJ, Campbell T, Burke KJ, Garcia B, Mlynarski A, et al. (2016) Synthesis and Characterization of a Chitosan/PVA Antimicrobial Hydrogel Nanocomposite for Responsive Wound Management Materials. J Microb Biochem Technol 8: 065-070. doi: 10.4172/19485948.1000264

of the substrate. The application of this ideology to current hydrogel technology can allow for the development of burn wound management materials with increased rigidity and sustainability.

This paper reports the synthesis and characterization of a nanocomposite chitosan/PVA hydrogel matrix coupled with photochemically prepared antimicrobial nanoparticles $\left(\mathrm{Ag} / \mathrm{TiO}_{2}\right)$. The addition of Ag-functionalized nanoparticles to the hydrogel matrix has shown to improve structural rigidity without compromising the rate of fluid uptake (i.e. swellabilty) as well provides a site for controlled release (i.e. no static ion leaching) of $\mathrm{Ag}^{+}$necessary for bacterial disinfection. Upon optimization of the synthetic parameters (i.e. balance of biopolymer and synthetic polymer) the nanocomposite has shown excellent biocompatibility with human dermal fibroblasts.

\section{Materials and Methods}

\section{Pre-coated Nanoparticle Synthesis}

Modification to a previously reported method were used to prepare Ag-functionalized $\mathrm{TiO}_{2}$ nanoparticles by irradiating suspended $\mathrm{TiO}_{2}$ in aqueous solution with ultraviolet light (UV) to reduce $\mathrm{Ag}^{+}$onto the $\mathrm{TiO}_{2}$ surface. Ethanol (ETOH) was added to the system to fill the electron hole and prevent charge recombination. Aeroxide ${ }^{\infty}$ anatase (80\%) $\mathrm{TiO}_{2}$ nanoparticles (Evonik Industries) with an approximate diameter of $25 \mathrm{~nm}$ were used as the core particle for all systems presented in this work. Nanoparticle dispersions, in water, were prepared by adding $0.1 \mathrm{wt} . \% \mathrm{TiO}_{2}$ and $1.0 \mathrm{wt} . \%$ ethanol to a $250 \mathrm{~mL}$ Pyrex flask. The resultant dispersion was ultrasonicated (Branson Instruments) for 30 minutes to break up any weak aggregates that may have occurred upon core nanoparticle addition. The post-sonication dispersion was transferred to a $500 \mathrm{ml}$ 3-neck Pyrex round bottom flask and placed in the photoreactor chamber (Luzchem) with an irradiation wavelength centered at $254 \mathrm{~nm}$. A second solution containing the desired concentration of $\mathrm{Ag}^{+}(1 \mathrm{mM}, 5 \mathrm{mM}, 10 \mathrm{mM})$ was prepared and placed into a separatory funnel fixed above the round bottom flask. The solution was allowed to slowly drip (approximately $3.0 \mathrm{~mL} / \mathrm{min}$ ) into the reaction vessel while exposed to UV light under constant nitrogen purge for 1 hour. After reaction, the particles were dialyzed for 48 hours using dialysis tubing with a molecular weight cutoff $12,000-14,000$ (Carolina Supplies) to remove any unreacted $\mathrm{Ag}^{+}$. Using Dynamic Light Scattering (DLS) (Malvern Instruments) the average particle size was determined (post-reaction/post-dialysis) for a representative sample $(3.0 \mathrm{ml})$ of photo-reacted nanoparticle dispersion. Additionally the isoelectric point was determined via $\mathrm{pH}$ sweeps (adjusted with $0.05 \mathrm{M}$ $\mathrm{HNO}_{3}$ and $0.1 \mathrm{M} \mathrm{NaOH}$ ) and the zeta potential (approximately $1 \mathrm{ml}$ sample) was measured at each condition using the Malvern Zetasizer (Malvern Instruments).

\section{Nanocomposite Hydrogel Synthesis}

Due to solubility limitations of both chitosan and PVA, 2.0 wt. $\%$ stock solutions were prepared in $4 \%$ aqueous acetic acid. The nanocomposite hydrogels were prepared by adding $0.01 \% \mathrm{TiO}_{2}$ as a substrate for the photo-reduction of $\mathrm{Ag}$ to varied ratios of the stock solutions (10:90, 15:85, and 20:80 chitosan:PVA) and treated in an ultrasonic bath (Branson Instruments) for 30 minutes to break up any aggregates. The chitosan solution was then added to the $\mathrm{PVA} / \mathrm{TiO}_{2}$ dispersion and stirred for 15 minutes. A $1 \mathrm{~mL}$ aliquot of glutaraldehyde was slowly added to the $\mathrm{PVA} /$ chitosan/ $\mathrm{TiO}$ mixture to promote the effective crosslinking of the nanocomposite hydrogel matrix [30,31]. Upon standing for 10 minutes, the gel-like dispersion was poured into a petri dish and allowed to set for 24 hours. The resultant gel was washed with $12 \% \mathrm{NaOH}$ followed by deionized water and dehydrated in a furnace at $140^{\circ} \mathrm{C}$ until dry.

After dehydration, gel samples are photo-reacted in-situ using $10 \mathrm{mM}$ silver nitrate $\left(\mathrm{AgNO}_{3}\right)$ with two different methods. The first method involves soaking the gel samples in $\mathrm{Ag}$ for 30 minutes and then photo-reacting for an additional 30 minutes. The second method involves soaking the gel samples in $\mathrm{AgNO}_{3}$ while simultaneously photo-reacting for 30 minutes under a constant a nitrogen $\left(\mathrm{N}_{2}\right)$ purge. The Ag coated gels are stored in a freezer to prevent from hardening.

\section{Swelling studies}

The fluid absorbing capacity (swellability) of the hydrogel is one of the important criteria for maintaining a moist wound environment. The initial mass of the as prepared hydrogel nanocomposite will be recorded and a $2 \mathrm{~cm}$ in size hydrogel disk will be placed in aqueous media. The hydrogel will be exposed to the fluid under different conditions ( $\mathrm{pH}$, time, temperature) that will attempt to mimic the physiological environment of the wound. The hydrogel absorbing capacity or degree of swelling (DOS) will be calculated using the following equation:

$$
D O S=\frac{W_{t}-W_{0}}{W_{0}} \times 100 \%
$$

where $\mathrm{W}_{0}$ and $\mathrm{W}_{\mathrm{t}}$ are the weights of the nanocomposite before exposure to buffer solution and the weight of the swollen nanocomposite. Observation of the DOS as a function of time at controlled temperature the equilibrium concentration as well as the rate of swellability can be determined.

\section{Antimicrobial testing}

Cultures of both E. coli (ATCC-25922) and S. aureus (ATCC-6538) were grown at $37^{\circ} \mathrm{C}$ in a standard tryptic soy broth (TSB) to an optical density $\left(\mathrm{OD}_{660}\right)$ of 0.5 . Each liquid bacterial culture was then aseptically swabbed onto a Mueller-Hinton (MH) Agar plate, and sections of each hydrogel (approximately $1.0 \mathrm{~cm}^{2}$ ) were then placed onto each plate. Following incubation for 18 hours at $37^{\circ} \mathrm{C}$, the zone of inhibition (ZOI) around each hydrogel was measured to quantify AME.

\section{Cell culture}

Adult human dermal fibroblasts (HDFa) were routinely maintained in $25 \mathrm{~cm}^{2}$ flasks in supplemented medium 106 with LSGS Kit (Invitrogen) containing $10 \mathrm{ml}$ of fetal bovine serum (FBS), $1.5 \mu \mathrm{g} / \mathrm{ml}$ recombinant human basic fibroblast growth factor $/ 5 \mathrm{mg} / \mathrm{ml}$ heparin, $1 \mathrm{mg} / \mathrm{ml}$ hydrocortisone, $1 \mathrm{ml}$ gentamicin/amphotericin $\mathrm{B}, 5.0 \mu \mathrm{g} / \mathrm{ml}$ recombinant human epidermal growth factor and kept at $37^{\circ} \mathrm{C}$ in a $5 \%$ $\mathrm{CO}_{2} / 95 \%$ air humidified incubator. Initial seeding was done following the determination of viable cell density. A seeding density of $5 \times 10^{3}$ viable cells $/ \mathrm{cm}^{2}$ was confirmed by combining $5 \mu$ of HDFa cells with $5 \mu \mathrm{l}$ of trypan blue (Invitrogen) into a hemacytometer. The contents of the cell vial $(1.0 \mathrm{ml})$ was diluted to a concentration of $2.5 \times 10^{4}$ viable cells $/ \mathrm{ml}$ using the supplemented medium $106.5 \mathrm{ml}$ of cell suspension was placed into four $25 \mathrm{~cm}^{2}$ flasks, swirled to distribute cells evenly and left undisturbed for 72 hours at $37^{\circ} \mathrm{C}$ in a $5 \% \mathrm{CO}_{2} / 95 \%$ air humidified incubator.

Culture medium was changed every other day until cells reached $80 \%$ confluency. At that time cells were subcultured by removing all medium from the flasks and rinsing with $5 \mathrm{ml}$ of Dulbecco's Phosphate Buffered Saline (DPBS, Invitrogen) without calcium and magnesium. Following removal of DPBS, $3 \mathrm{ml}$ of TRYPLE Express with phenol red (TrypLE) was added to flasks and placed in incubator for 10 minutes 
Citation: Rinehart SJ, Campbell T, Burke KJ, Garcia B, Mlynarski A, et al. (2016) Synthesis and Characterization of a Chitosan/PVA Antimicrobial Hydrogel Nanocomposite for Responsive Wound Management Materials. J Microb Biochem Technol 8: 065-070. doi: 10.4172/19485948.1000264

to remove cells. After all cells were dislodged, $5 \mathrm{ml}$ of medium 106 was added to flasks and the entire volume was collected in a $50 \mathrm{~mL}$ conical tube. Cell suspension was spun at $100 \times \mathrm{g}$ for 9 minutes forming a cell pellet. The supernatant was discarded and the cells were re-suspended with fresh medium 106. Concentration of viable cells was determined; cells were diluted and placed in new $25 \mathrm{~cm}^{2}$ flasks.

After four successful passages, cells were subcultured (above) and placed into a flask that contained one of twenty-four different unlabeled biomimetic hydrogels or no hydrogel (control). This was repeated 3 times for each hydrogel. The flasks were returned to the incubator and left undisturbed for 24 hours. After 24 hours, cell growth was examined using a Nikon Eclipse TS100 inverted fluorescent microscope. A live/ dead cell imaging kit 488/570 (Molecular Probes, Invitrogen) was used to assess cell viability in the flask and in proximity to the hydrogel. Equal amounts of Live Green and Dead Red vials were added to an equal amount of cells and incubated for 15 minutes.

\section{Results and Discussion}

\section{Effect of Reaction pH on Ag-functionalized Nanoparticle Size}

Synthetic modulation of the Ag-functionalized nanoparticle size is a critical attribute with respect to hydrogel matrix stability and the overall AME. It is believed the size of the nanoparticle will play a major role in the hydrogen-bonding network formation that is essential for the hydrogel matrix to exhibit the desired structural properties as well as the ability to achieve the desired swelling efficiency. Previous work has also suggested that smaller nanoparticles exhibit enhanced antimicrobial properties as a function of the effective surface area available for bacteria cell interactions. Figure 1 is a summary of the effect of reaction $\mathrm{pH}$ on the size of the Ag-functionalized nanoparticle after photo-deposition and post-dialysis. As pre-reaction $\mathrm{pH}$ of the dispersion increases the surface charge of the core $\mathrm{TiO}_{2}$ nanoparticle approaches its soelectric point (measured in reaction mixture to be $\mathrm{pH}=5.3$ ), which results in the charge destabilization of the core particle and the subsequent formation of aggregates of the functionalized

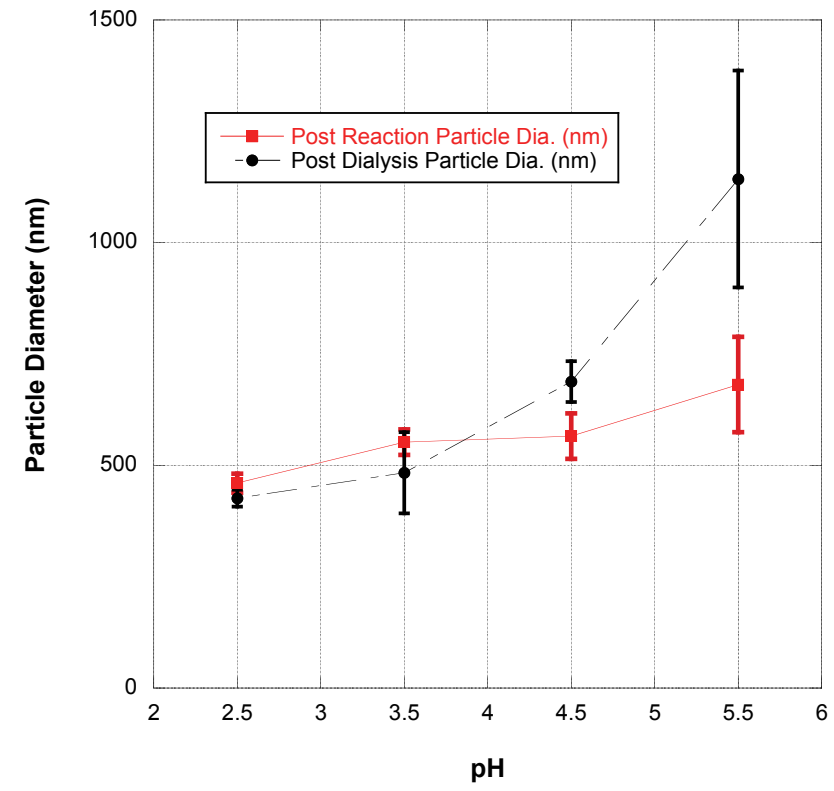

Figure 1: Effect of reaction $\mathrm{pH}$ and dialysis on Ag-functionalized nanoparticle size. particle. At lower $\mathrm{pH}$ there is significant charge repulsion of the core particles, which modulates the degree of aggregation and results in lower average particle size post- reaction. While lower $\mathrm{pH}$ is desired for the formation of smaller post-reaction particles the ability of the deposited $\mathrm{Ag}^{0}$ to undergo dissolution to $\mathrm{Ag}^{+}$in the acidic media is possible. In order to control this effect the post-reaction dispersion underwent dialysis (48-hours) in neutral $\mathrm{pH}=6.0$ deionized water to neutralize the acidic environment and quench the ability of the deposited $\mathrm{Ag}^{0}$ to ionize. Figure 1 clearly indicates that the $\mathrm{pH}=2.5$ reaction condition shows little deviation between the post-dialysis and post-reaction particle size. Validation of the aggregation effect can be seen at the $\mathrm{pH}$ of 5.5 condition where there is severe aggregation of the particle post-dialysis indicating reduced Ag surface coverage that results in the ability of the core nanoparticles to form large aggregates.

\section{Effect of $\left[\mathrm{Ag}^{+}\right]$on Ag-functionalized Nanoparticle Size}

Using the desired reaction conditions $(\mathrm{pH}=2.5$ and 48-hour dialysis) the effect of pre-reaction $\mathrm{Ag}^{+}$concentration was evaluated to determine its effect on particle size and the isoelectric point of the functionalized particle. The results indicate that the particle size increases slightly upon the deposition of $1 \mathrm{mM} \mathrm{Ag}$ and trends upward as a function of concentration ( 5 and $10 \mathrm{mM}$ ). This clearly shows that upon irradiation in the presence of $\mathrm{Ag}^{+}$the surface of the core $\mathrm{TiO}$ nanoparticle is being modified. As increased amounts of $\mathrm{Ag}^{+}$are added to the reaction mixture there is apparent surface saturation reached which may result in a slight increase of postreaction particle aggregation. To further validate Ag-functionalization on the surface of the core $\mathrm{TiO}_{2}$ nanoparticles the isoelectric point of the post-dialysis dispersions were measure and compared against a naked $\mathrm{TiO}_{2}$ nanoparticle. The results in Table 1 validate that as deposition onto the surface of the core nanoparticle occurs there is a shift in the isoelectric point as a function $\mathrm{Ag}$ concentration increased. Adding $\mathrm{Ag}$ to the surface of $\mathrm{TiO}_{2}$ changes the surface structure of the core particle, which results in the observed isoelectric point shifts. Additionally these results support the surface saturation hypothesis at $5 \mathrm{mM}$ because there is a minimal isoelectric point shift demonstrating no further Ag was deposited. Based on this result it was determined that the optimal $\left[\mathrm{Ag}^{+}\right]$for particle size balance is centered on the $5 \mathrm{mM}$ region.

\section{Antimicrobial Efficiency (AME) of Ag-functionalized Nanoparticles}

Disk diffusion tests were conducted using the optimal synthetic pathway to determine the effect of pre-reaction $\left[\mathrm{Ag}^{+}\right]$on the effective ZOI. As previously stated, Ag-functionalized nanoparticle samples were pressed into uniform pellets, placed over a bacteria-coated plate, and incubated for 18 hours. Figure 2 is a summary of the ZOI for cultures of E. coli and S. aureus as a function of Ag concentration. It must be noted that the functionalized nanoparticle size for the various concentrations remained relatively constant as to decouple the size and concentration effects. Against both bacteria, there appears to be a clear increase in the ZOI from the naked nanoparticle versus the addition of $1.0 \mathrm{mM} \mathrm{Ag}$ to the particle. This is further validation of the claim that

\begin{tabular}{|c|c|c|}
\hline$\left[\mathrm{Ag}^{+}\right](\mathbf{m M})$ & $\begin{array}{c}\text { Average Particle Size } \\
(\mathbf{n m})\end{array}$ & Isoelectric Point $(\mathbf{p H})$ \\
\hline $0 "$ & $292 " \pm " 15 "$ & $5.3 " \pm " 0.2 "$ \\
\hline $1 "$ & $344 " \pm " 41 "$ & $5.5 " \pm " 0.3 "$ \\
\hline $5 "$ & $397 " \pm " 28 "$ & $6.3 " \pm " 0.1 "$ \\
\hline $10 "$ & $443 " \pm " 31 "$ & $6.4 " \pm " 0.2 "$ \\
\hline
\end{tabular}

Table 1: Average particle size and isoelectric point as a function of $\mathrm{Ag}^{+}$concentration during deposition on to $\mathrm{TiO}_{2}$. 
Citation: Rinehart SJ, Campbell T, Burke KJ, Garcia B, Mlynarski A, et al. (2016) Synthesis and Characterization of a Chitosan/PVA Antimicrobial Hydrogel Nanocomposite for Responsive Wound Management Materials. J Microb Biochem Technol 8: 065-070. doi: 10.4172/19485948.1000264

the addition of Ag results in the effective inhibition of bacterial growth. As the concentration of the Ag increased, there was no increase in the effective ZOI, which indicates the functionalized nanoparticle contains the necessary surface concentration to effectively inhibit the growth of both E. coli and S. aureus. It should be noted that this concentration occurred around the $5.0 \mathrm{mM}$ region, thus matching the optimal concentration for particle size control.

\section{Optimization of PVA/Chitsoan Nanocomposite Hydrogel Degree of Swelling}

There has been a significant amount of work conducted to describe the kinetic and thermodynamic of the fluid uptake (swelling) of various hydrogel systems. Swelling has been described as the transition from an unsolvated state to a state where the pores of the hydrogel nanocomposite are swollen (solvated) with fluid. More specifically this has been reported as the diffusion of solution into the polymeric matrix of the hydrogel resulting in expansion and retention of the external fluid. This work adds an extra level of complexity to the swelling process via the incorporation of functionalized nanoparticles within the polymeric matrix. The hydrogel nanocomposite system was optimized by evaluating the ratio of biopolymer (chitosan) and swelling agent (PVA) in the presence of core $\mathrm{TiO}_{2}$ nanoparticles to determine the concentration that produce maximum swell capacity without compromising the structural integrity of the nanocomposite. Figure 3 is the summary of the effect of additive ratios at fixed nanoparticle concentration $(0.1 \mathrm{wt} \%)$ on the overall degree of swelling. The results clearly show the balance needed to achieve maximum swelling requires a high level of PVA to ensure effective hydrogen bonding in the hydrogel matrix. The addition of increased level of chitosan created a more fiber like structure, which resulted in a reduced degree of swelling and furthermore comprised the structural integrity of the gel. The final phase of optimization was to explore the effect of the Ag-functionalization method (pre-coated vs. in-situ) on the degree of swelling. Figure 4 shows the effect of deposition method on the degree

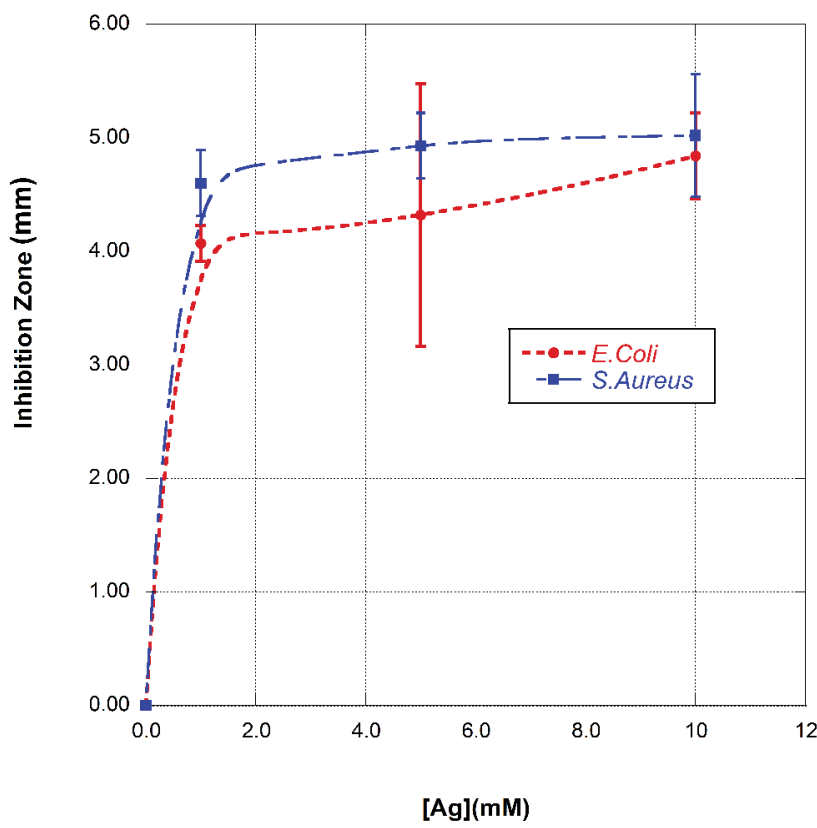

Figure 2: Zone of inhibition for both $E$. coli and S. aureus during Antimicrobial efficiency testing as a function of $\mathrm{Ag}^{+}$concentration.

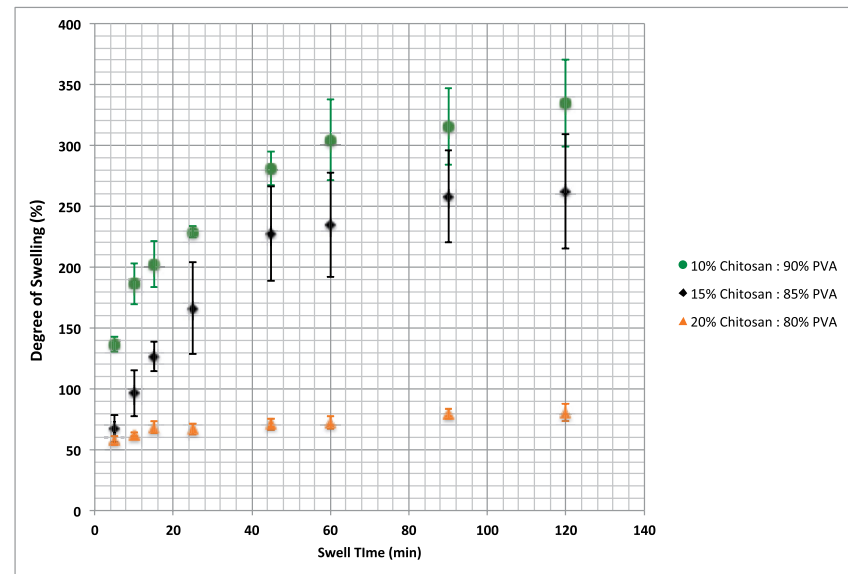

Figure 3: Effect Chitosan/PVA concentrations on nanocomposite degree of swelling.

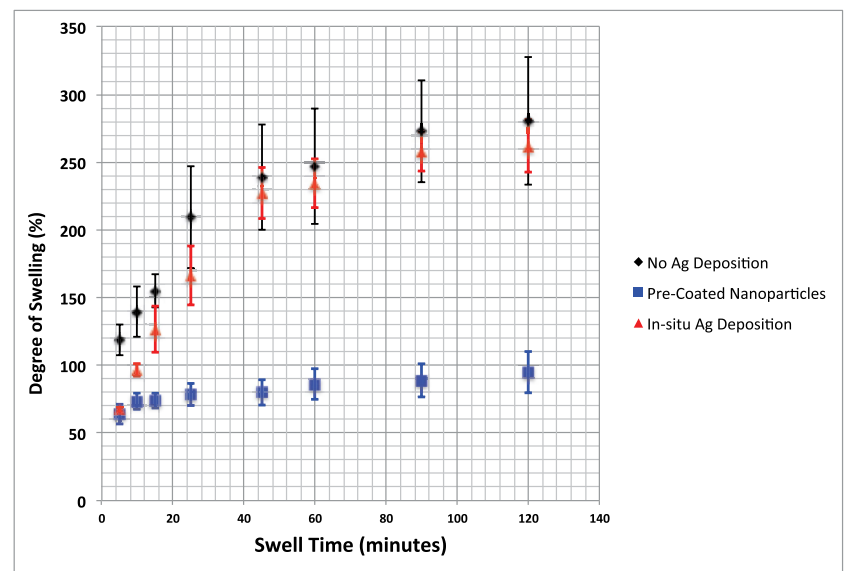

Figure 4: Effect $\mathrm{Ag}^{+}$deposition methods on nanocomposite degree of swelling

of swelling for the optimized hydrogel synthesis pathway ( $\mathrm{pH}=2.5$ and 5 $\mathrm{mM} \mathrm{Ag}$ ). It is clear that the addition of Ag-functionalized nanoparticles using the in-situ deposition method produced swelling effectiveness similar to that of the unmodified core particle. The preparation of hydrogel nanocomposites using the pre-coated nanoparticles showed a significant decrease in overall swelling effectiveness. This decrease is attributed to the reduction of effective incorporation of the functionalized nanoparticle into the hydrogel matrix. More specifically shifting the surface energy (hydrophobic/hydrophilic interactions) of the particle resulted in a reduction of effective crosslinking (hydrogen bonding) that impacted the structural integrity of the gel.

\section{Antimicrobial Effectiveness of Optimized Hydrogel Nanocomposite}

Figure 5 is a summary of the ZOI for cultures of E. coli and $S$. aureus for the various methods of nanoparticle functionalization using the optimized synthesis parameters (15\% PVA / 85\% Chitosan and $5 \mathrm{mM}[\mathrm{Ag}])$. These data suggest that either method of nanoparticle functionalization will provide effective inhibition of bacterial growth, although it is proposed that the greatest inhibition can be obtained 
Citation: Rinehart SJ, Campbell T, Burke KJ, Garcia B, Mlynarski A, et al. (2016) Synthesis and Characterization of a Chitosan/PVA Antimicrobial Hydrogel Nanocomposite for Responsive Wound Management Materials. J Microb Biochem Technol 8: 065-070. doi: 10.4172/19485948.1000264

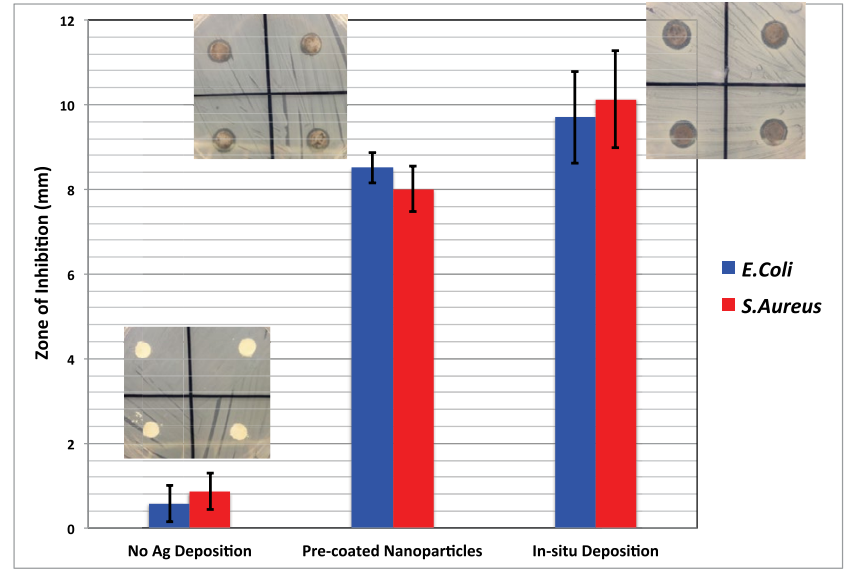

Figure 5: Effect of $\mathrm{Ag}^{+}$deposition method on antimicrobial efficiency of both $E$. coli and S. aureus.

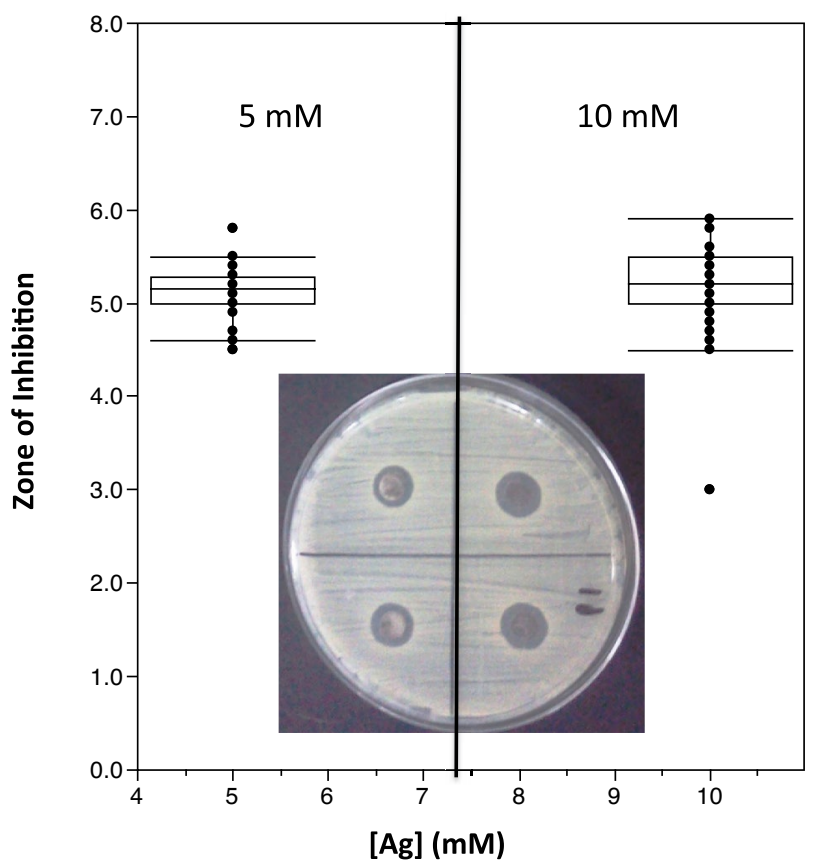

Figure 6: Antimicrobial Efficiency of optimized nanocomposite on MRSA as a function of $\mathrm{Ag}^{+}$concentration.

via the in-situ method of deposition. This may be related to the ability of the particle to be functionalized uniformly through the depth of the hydrogel matrix and thus would have an impact on the effective transport of the necessary $\mathrm{Ag}^{+}$to the bacterial cell wall. Additionally this can be coupled with the fact that the structural integrity of the hydrogel produced using the in-situ method as well as its effective degree of swelling results in more efficient ion transport to result in cell lysis. Further validation of the effective antimicrobial properties of the optimized hydrogel nanocomposite can be seen in Figure 6. A culture of Methicillin-resistant Staphylococcus aureus (MRSA) was exposed for 18 hours to the optimized hydrogel nanocomposite at two levels (5.0 and $10.0 \mathrm{mM}$ ) of in-situ Ag-functionalization [29]. The ZOI results clearly show effective inhibition of MRSA growth indicating the nanocomposite hydrogels effectiveness in the presence of a clinically relevant multi-drug resistant bacterial species.

\section{Effect of Optimized Hydrogel Nanocomposite on Adult Human Dermal Fibroblast (HDFa) Growth}

Figure 7 is a representative summary of adult human dermal fibroblast (HDFa) growth in the presence of different ratios of additives (PVA/Chitosan) with constant in-situ functionalized nanoparticle concentration ( $5 \mathrm{mM} \mathrm{Ag}$ with 0.1 wt.\% core particle). HDFa were grown in culture containing a biomimetic hydrogel constructed of either 50\% PVA / 50\% Chitosan (A), 10\% PVA / 90\% Chitosan (B), 20\% PVA / 80\% Chitosan (C), and 15\% PVA / 85\% Chitosan (D). Fibroblasts with a bipolar appearance that are dispersed evenly on the flask bottom demonstrate successful cell growth (B-D), whereas clumped circular cells are evidence of cell death (A). To further investigate cell viability, a live (green)/ dead (red) protein stain was added in culture. Figure 7 shows significantly higher cell death in the presence of higher concentration of PVA (A). In addition, the ability of human dermal fibroblasts to proliferate on and adjacent to varying hydrogels was determined. Photomicrographs taken of HDFa in culture showed proliferating HDFa growth directly below and adjacent to hydrogels optimized to 15\% PVA / 85\% Chitosan (Figure 8). Flasks containing a higher concentration of PVA either inhibited fibroblast growth completely or impeded growth near the hydrogel.

\section{Conclusion}

This work developed a photochemical method to prepare $\mathrm{Ag}^{+}$functionalized $\mathrm{TiO}_{2}$ nanoparticles at a controlled particle size and observe the resulting antimicrobial activity $E$. coli and $S$. aureus. A synthetic protocol was developed implementing a pre-reaction sonication and slow-drip method to minimize the amount of aggregates formed during the photo- reduction process. The effect of reaction $\mathrm{pH}$ and $\mathrm{Ag}$ concentration on the overall particle size and antimicrobial effectiveness was studied. Furthermore, the antimicrobial effect of both nanocomposites and nanoparticle dispersions was observed. The antimicrobial activity of the prepared nanocomposites clearly shows

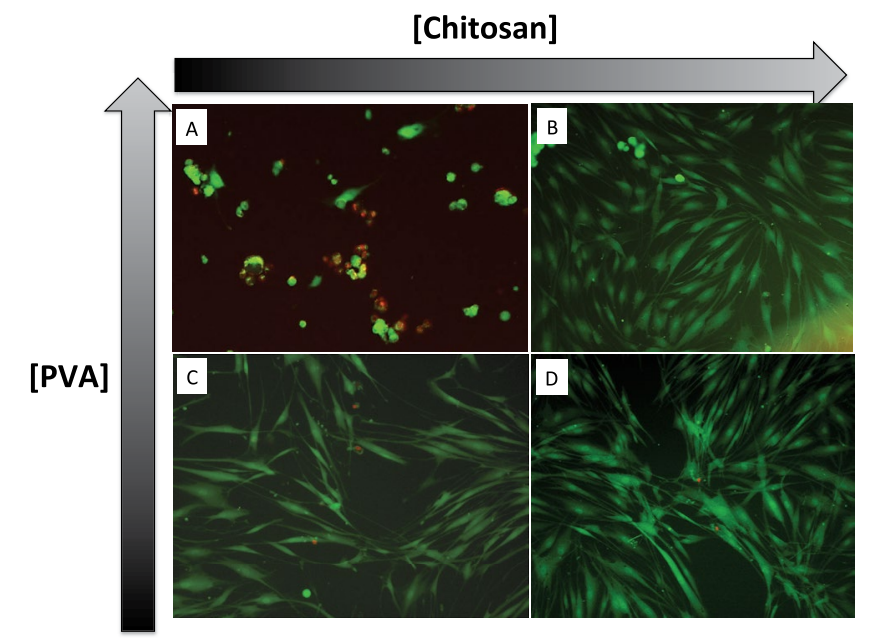

Figure 7: Adult Human dermal fibroblast growth (HDFa) photomicrographs (10X magnification) in culture using live (green) / dead (red) cell imaging kit 488 / 570 (Molecular Probes, Invitrogen). HDFa grown in flasks containing a biomimetic hydrogel constructed of 50\% PVA / 50\% Chitosan (A), 10\% PVA / 90\% Chitosan (B), 20\% PVA / 80\% Chitosan (C), and 15\% PVA / 85\% Chitosan (D). 
Citation: Rinehart SJ, Campbell T, Burke KJ, Garcia B, Mlynarski A, et al. (2016) Synthesis and Characterization of a Chitosan/PVA Antimicrobial Hydrogel Nanocomposite for Responsive Wound Management Materials. J Microb Biochem Technol 8: 065-070. doi: 10.4172/19485948.1000264

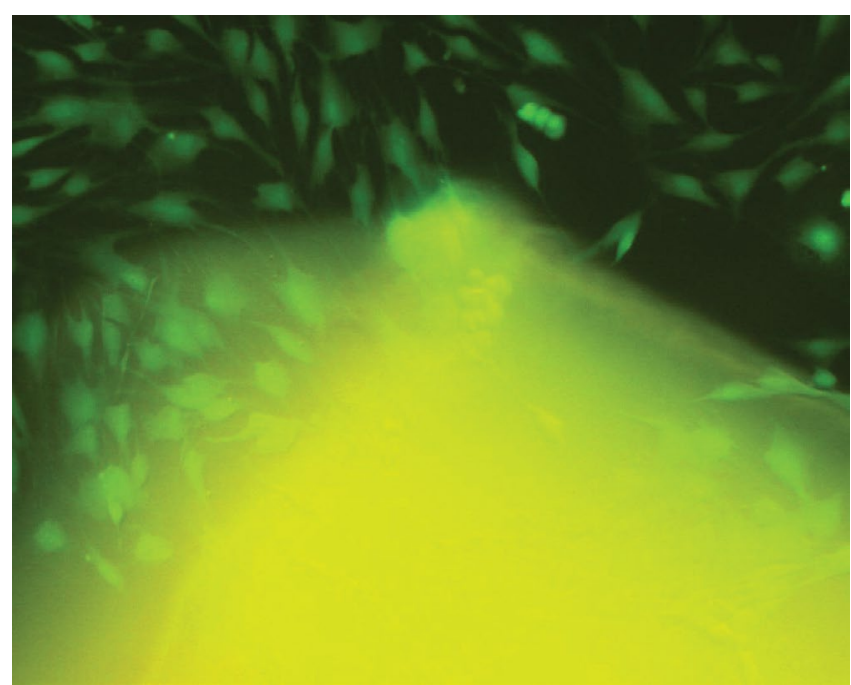

Figure 8: Adult human dermal fibroblast growth (HDFa) photomicrograph (20X magnification) showing successful cell growth adjacent to and directly under the biomimetic hydrogel.

that there is inhibited bacterial growth. It was also determined that the method of hydrogel synthesis (i.e. Ag deposition process) has an impact on the performance of the nanocomposite. Furthermore the fibroblast growth occurred directly below the hydrogel on the flask bottom, demonstrating that if this hydrogel were applied to a wound, cell growth would not be impeded. These results reveal that when PVA increases in the system, there is no appreciable cell growth. On the other hand, when the ratio of biomimetic chitosan and PVA is optimized to reduce the overall amount of synthetic polymer, there is healthy cell growth. This result directly speaks to a key finding that reveals the importance of the correct balance or the removal of synthetic polymer from the system.

\section{Acknowledgements}

This work was supported by grants from the Colonel Stephen S. and Lyla Doherty Center for Aviation and Health Research at Lewis University. The authors would to thank Daniel Turner, Michael Hegazin, Steven Neubauer, and James Fitman for the their initial work on the development of the Ag-nanoparticle synthesis pathway as well Travis Buckley and Daniel Ryan for their support with respect to antimicrobial testing.

\section{References}

1. Abdeen Z (2001) Swelling and Reswelling Characteristics of Cross-Linked Poly (vinyl alcohol) / Chitosan Hydrogel Film. J Dispers Sci Technol 201: 1337-1344.

2. Ngamviriyavong $P$, Thananuson $A$, Pankongadisak $P$, Tanjak $P$, Janvikul $W$ (2010) Antibacterial Hydrogels from Chitosan Derivatives. Chem 20: 113-117.

3. Wang X, Bai H, Yao Z, Liu A, Shi G (2010) Electrically conductive and mechanically strong biomimetic chitosan/reduced graphene oxide composite films. J Mater Chem 20: 9032.

4. Zhou Y, Ma G, Shi S, Yang D, Nie J (2011) Photopolymerized water-soluble chitosan-based hydrogel as potential use in tissue engineering. Int $\mathrm{J}$ Biol Macromol 48: 408-413.

5. Saha N (2001) Polymeric Biomaterial Based Hydrogels for Biomedical Applications. J Biomater Nanobiotechnol 201: 85-90.

6. Valmikinathan C, Bellamkonda R (2010) Photo-Crosslinkable Chitosan Based Hydrogels for Tissue Engineering. Technology. Chem 1: 86-330.

7. Varshosaz J, Koopaie N (2001) Cross-linked poly (vinyl alcohol) hydrogel: study of swelling and drug release behaviour. Iran Polym J 1: 123-131.
8. Schwall CT, Banerjee I (2009) Micro- and Nanoscale Hydrogel Systems for Drug Delivery and Tissue Engineering 2: 577-612.

9. Khor E, Wu H, Lim L (2001) Preparation, Characterization and Hydroge Formation. Materials (Basel) 201: 1728-1746.

10. Sannino A, Demitri C, Madaghiele M (2009) Biodegradable Cellulose-based Hydrogels: Design and Applications. Materials (Basel) 4: 353-373.

11. Riva R, Ragelle H, Rieux A (2001) des. Chitosan and chitosan derivatives in drug delivery and tissue engineering. Adv Polym Sci 24: 19-44.

12. Agnihotri S, Mukherji S, Mukherji S (2012) Antimicrobial chitosan-PVA hydrogel as a nanoreactor and immobilizing matrix for silver nanoparticles. Appl Nanosci 2: 51-59.

13. Hennink WE (2001) Novel crosslinking methods to design hydrogels. Adv Drug Deliv Rev 6: 223-236.

14. Yang X, Zhu Z (2008) Effects of PVA, agar contents, and irradiation doses on properties of PVA/ws-chitosan/glycerol hydrogels made by -irradiation followed by freeze-thawing. Radiat Phys Chem 77: 954-960.

15. Selvarani M, Prema P (2001) Evaluation of Antibacterial Efficacy of Chemically Synthesized Copper and Zerovalent Iron Nanoparticles. Asian J Pharm Clin Res 2: 6.

16. Stephens-Altus JS, Sundelacruz P, Rowland ML, West JL (2011) Development of bioactive photo-cross-linkable fibrous hydrogels. J Biomed Mater Res A 98 167-176.

17. Naylor WA1 (2005) A guide to wound managment in palliative care. Int J Palliat Nurs 11: $57,574-579$.

18. Rathinamoorthy R, Sasikala L (2001) Polysaccharide Fibers in Wound Management. Int J Pharm Sci 201: 38-44.

19. Nacer Khodja A, Mahlous M, Tahtat D (2001) Evaluation of healing activity of PVA / chitosan hydrogels on deep second degree burn: pharmacological and toxicological tests. Burns 39: 98-104.

20. Jayakumar R, Prabaharan M (1990) Novel Chitin and Chitosan Materials in Wound Dressing. Langmuir 3: 24

21. Petrulyte $S$ (2008) Advanced textile materials and biopolymers in wound management. Dan Med Bull 55: 72-77.

22. Ferraria AM, Boufi S, Battaglini N, Botelho do Rego AM, ReiVilar M (2010) Hybrid systems of silver nanoparticles generated on cellulose surfaces. Langmuir 26: 1996-2001.

23. Ahmadi F, Abolghasemi S, Parhizgari N (2001) Effect of Silver Nanoparticles on Common Bacteria in Hospital Surfaces. Jundishapur J Microbiol 6: 209-215.

24. Honary S, GhajarK, Khazaeli P, Shalchian P(2001)Preparation, Characterization and Antibacterial Properties of Silver-Chitosan Nanocomposites Using Different Molecular Weight Grades of Chitosan. Trop J Pharm Res 10: 69-74.

25. Warriner R, Burrell R (2005) Infection and the chronic wound: a focus on silver Adv Skin Wound Care 18 Suppl 1: 2-12

26. Vimala K (2001) Fabrication of Curcumin Encapsulated Chitosan-PVA Silver Nanocomposite Films for Improved Antimicrobial Activity. J Biomater Nanobiotechnol 1: 55-64

27. Keleher J, Bashant J, Heldt N (2001) Photo-catalytic preparation of silver-coated $\mathrm{TiO}_{2}$ particles for antibacterial applications. World Journal of Microbiology and Biotechnology 200: 133-139.

28. Ocwelwang AR, Tichagwa $L$ (2001) Synthesis and Characterisation of $A g$ and Nitrogen Doped Tio2 Nanoparticles Supported on A Chitosan-Pvae Nanofibre Support 201: 28-37.

29. Rago JV, Buhs LK, Makarovaite V, Patel E, Pomeroy M, et al. (2012) Detection and analysis of isolates found in ambulances in the Chicago metropolitan area. Am J Infect Control 40: 201-205.

30. Jin L, Bai R (2001) Mechanisms of Lead Adsoprion on Chitosan / PVA Hydroge Beads. Langmuir 25: 9765-9770.

31. Schiffman JD, Schauer CL (2007) Cross-linking chitosan nanofibers. Biomacromolecules 8: 594-601. 